\title{
Estatus epiléptico refractario no convulsionante en postoperatorio de pancreatectomía total: A propósito de un caso
}

García Martínez JA, Benimeli López MC, García Legaz Navarro F, García Palenciano C.

Hospital Clínico Universitario Virgen de la Arrixaca de Murcia.

\section{Resumen}

Se presenta un caso clínico de Estatus Epiléptico No convulsionante en el postoperatorio de una paciente de 79 años sin antecedentes de epilepsia, y su probable relación con la antibioterapia pautada. Seguidamente se realiza una discusión acerca del estatus epiléptico y su aparición como efecto secundario del tratamiento con ciertos antibióticos.

\section{Introducción}

Se presenta un caso clínico de Estatus Epiléptico No convulsionante en el postoperatorio de una paciente de 79 años sin antecedentes de epilepsia, y su probable relación con la antibioterapia pautada. Seguidamente se realiza una discusión acerca del estatus epiléptico y su aparición como efecto secundario del tratamiento con ciertos antibióticos.

El estatus epiléptico se define como una crisis epiléptica prolongada o una serie de crisis durante las cuales el paciente no recobra completamente la conciencia. Actualmente se acepta como estatus epiléptico cualquier actividad epiléptica de más de 5 minutos de duración, caracterizada por una crisis duradera, o dos o más crisis durante las cuales el paciente no retorna a su situación previa de conciencia.

Algunos cursan con convulsiones aparentes, mientras que otros pueden pasar de manera completamente inadvertidos. Esto resulta de interés a la hora de establecer un diagnóstico diferencial ante un deterioro del nivel de conciencia/falta de recuperación de la misma sin causa orgánica que lo justifique.

\section{Caso Clínico}

Paciente mujer de 79 años de edad con antecedentes de Hipertensión Arterial. Tres días antes de la intervención la paciente desarrolla un cuadro clínico compatible con una colangitis aguda secundaria al proceso tumoral, motivo por el cual los servicios de Medicina Interna e Infecciosas deciden pautar Imipenem y Teicoplanina.

Es intervenida de neoplasia pancreática. Se realiza antrectomía, duodenopancreatectomía total con esplenectomía. La cirugía transcurre sin incidencias y es ingresada en la Unidad de Reanimación en ventilación espontanea, hemodinámicamente estable, consciente, orientada y sin focalidad neurológica. Manteniéndose a $\mathrm{su}$ ingreso el tratamiento antibiótico pautado. 
En el segundo del día del posoperatorio comienza con un cuadro de deterioro neurológico con tendencia a la somnolencia y estupor, febrícula y leucocitosis reactiva, planteándose un probable cuadro séptico como causa subyacente.

Ante esta situación se extraen nuevos cultivos de sangre, líquido biliar y lecho de la herida quirúrgica. Se pauta soporte respiratorio con gafas nasales de alto flujo y se solicita TAC de Abdomen/Tórax con contraste de manera urgente.

En el TAC se aprecia trombosis portal extensa, pero sin datos analíticos $\mathrm{o}$ clínicos de disfunción hepática y con valores de amonio posteriores normales, por lo que se instaura tratamiento anticoagulante. Ante la ausencia de signos o síntomas que hiciesen sospechar un cuadro meningítico agudo, se solicita un TAC cerebral, para descartar causa orgánica que justificara el estado neurológico, no encontrándose en el mismo, lesión que se correlacione con el estado de la paciente.

Al tercer día del posoperatorio, se aísla en cultivo de líquido biliar $\mathrm{C}$. Parapsilosis, sensible a Fluconazol, motivo por lo que se añade éste al tratamiento.

A pesar del tratamiento, la paciente permanece en un estado comatoso, con nula o puntual apertura ocular ante el dolor, emisión aislada de sonidos incomprensibles y sin ninguna respuesta motora apreciable. En ventilación espontanea con gafas nasales de alto flujo, buena mecánica respiratoria $\mathrm{y}$ correcto intercambio gaseoso.

Se solicita electroencefalograma urgente. Durante su realización, se aprecian descargas generalizadas de ondas agudas y puntas, a lo largo de todo el trazado, de una amplitud de hasta $160 \mu \mathrm{V}$ y con una frecuencia que oscila entre los 2-3,5 Hz, con mayor expresión en regiones frontotemporales. Esta actividad bioeléctrica anómala durante el registro es compatible con un patrón estatus epiléptico no convulsionante.

Frente a los nuevos hallazgos se inicia tratamiento con Levetiracetam, se retira Imipenem, por posible agente desencadenante, siendo sustituido por Meropenem, manteniéndose la Teicoplanina y el Fluconazol. Dada la falta de respuesta al Levetiracetam, el caso fue reevaluado en colaboración con el servicio de Neurología. Con la administración de Fenitoína la paciente recobro el estado normal de conciencia.

\section{Discusión}

El estatus epiléptico puede suponer una emergencia vital, llegando alcanzar una mortalidad del $50 \%$ en los casos refractarios a tratamientos de primera o de segunda línea, si no se practica atención inmediata. Es una entidad, cuyo pronóstico se ve fuertemente condicionado por la edad, duración, etiología, tipo de estatus y la respuesta o ausencia al tratamiento.

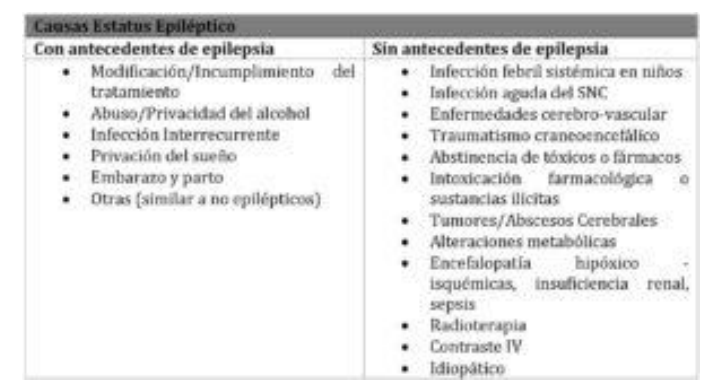

Tabla 1: Causas Estatus Epiléptico (Capítulo 6.3 Manual Medicina Intensiva $4^{\circ}$ Edición)

A diferencia del estatus epiléptico convulsionante, que requieren un tratamiento enérgico y rápido, el estatus epiléptico no convulsionante o larvado requiere de una confirmación electroencefalográfica para su diagnóstico. 


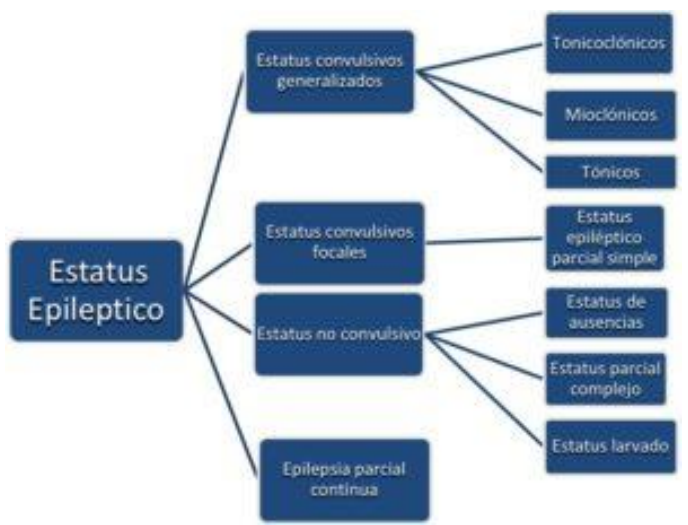

Diagrama 1: Clasificación diferentes estatus epilépticos

En los últimos años, se ha promovido el uso del Levetiracetam como fármaco de primera línea ante estatus epilépticos, debido sobre todo a la ausencia de efectos secundarios notables, su buena biodisponibilidad, la no interacción con receptores GABA o glutamato y su presentación intravenosa para su rápida difusión (1). Todo esto ha permitido relegar fármacos, que antiguamente eran de primera línea, a un segundo o tercer plano $\mathrm{o}$ ante situaciones de refractariedad.

Ahro Kim et al señalan en su artículo que: el estatus epiléptico no convulsionante puede ser una complicación neurológica asociada a la terapia con Cefepima, siendo su incidencia mayor en aquellos pacientes con insuficiencia renal asociada (2).

Dicho perfil neurotóxico, está presente en otros tipos de betalactámicos, como el Imipenem/Cilastatina, el cual presenta limitaciones a la hora de su utilización, por el alto riesgo de aparición de complicaciones neurológicas cuando es necesario su asociación con otros fármacos neurotóxicos de manera concomitante (3). A la hora de precisar un carbapenémico, Norrby SR y Gildon KM publicaron el mejor perfil farmacodinámico, la mejor tolerancia por parte del paciente y la menor incidencia de efectos adverso del Meropenem en su estudio (5).

\section{Bibliografía}

- Herrero Meseguer J.I., Corral Ansa L. Convulsiones. En: Montejo J.C., Garcia de Lorenzo A., Marco P., Ortiz C. Manual de Medicina Intensiva. $5^{\text {a }}$ ed. Barcelona: Elsevier; 2016. p. 212-217.

- Ahro Kim, Ji-Eun Kim, Young-Min Paek, Keun-Sik Hong, Young-Jin Cho, Hee-Kyung Park. Cefepime-Induced Non-Convulsive Status Epilepticus. J Epilepsy Res. 2013 Jun; 3(1): 3941 (ㅍML) $(\underline{\mathrm{PDF}})$

- Lizasoaín M., Noriega AR. Tolerance and safety of carbapenems: the use of meropenem. Enferm Infecc Microbiol Clin. 1997 Sep;15 Suppl 1:73-7. (PubMed)

- Geroulanos SJ. Meropenem versus imipenem/cilastatin in intra-abdominal infections requiring surgery. $\mathrm{J}$ Antimicrob Chemother. 1995 Jul;36 Suppl A:191-205. (PubMed)

- Norrby SR, Gildon KM. Safety profile of Meropenem: A review of nearly 5,000 patients treated with Meropenem. Scand J Infect Dis 1999 31(1):3-10. (PubMed)

- Navarro V, Mazoit JX. Drugs for status epilepticus treatment. Rev Neurol (Paris). 2009; 165:355-65. (PubMed)

Correspondencia al autor

José Antonio García Martínez

josgarmar007@gmail.com

MIR en Anestesiología, Reanimación y Terapéutica del Dolor.

Hospital Clínico Universitario Virgen de la Arrixaca de Murcia

Aceptado para blog en julio de 2018. 